\title{
Universal Behavior of Charged Particle Production in Heavy Ion Collisions at RHIC Energies
}

\author{
Peter A. Steinberg ${ }^{2}$ for the PHOBOS collaboration \\ B.B.Back ${ }^{1}$, M.D.Baker ${ }^{2}$, D.S.Barton ${ }^{2}$, R.R.Betts ${ }^{6}$, M.Ballintijn $^{4}$, A.A.Bickley ${ }^{7}$, R.Bindel ${ }^{7}$, A.Budzanowski ${ }^{3}$, W.Busza ${ }^{4}$, \\ A.Carroll $^{2}$, M.P.Decowski ${ }^{4}$, E.García ${ }^{6}$, N.George ${ }^{1,2}$, K.Gulbrandsen ${ }^{4}$, S.Gushue ${ }^{2}$, C.Halliwell ${ }^{6}$, J.Hamblen ${ }^{8}$, \\ G.A.Heintzelman ${ }^{2}$, C.Henderson ${ }^{4}$, D.J.Hofman ${ }^{6}$, R.S.Hollis ${ }^{6}$, R.Hołyński ${ }^{3}$, B.Holzman ${ }^{2}$, A.Iordanova ${ }^{6}$, E.Johnson ${ }^{8}$, \\ J.L.Kane ${ }^{4}$, J.Katzy ${ }^{4,6}$, N.Khan ${ }^{8}$, W.Kucewicz ${ }^{6}$, P.Kulinich ${ }^{4}$, C.M.Kuo ${ }^{5}$, W.T.Lin ${ }^{5}$, S.Manly ${ }^{8}$, D.McLeod ${ }^{6}$, \\ J.Michałowski $^{3}$, A.C.Mignerey ${ }^{7}$, R.Nouicer ${ }^{6}$, A.Olszewski ${ }^{3}$, R.Pak ${ }^{2}$, I.C.Park ${ }^{8}$, H.Pernegger ${ }^{4}$, C.Reed ${ }^{4}$, L.P.Remsberg ${ }^{2}$, \\ M.Reuter ${ }^{6}$, C.Roland ${ }^{4}$, G.Roland ${ }^{4}$, L.Rosenberg ${ }^{4}$, J.Sagerer ${ }^{6}$, P.Sarin ${ }^{4}$, P.Sawicki ${ }^{3}$, W.Skulski ${ }^{8}$, S.G.Steadman ${ }^{4}$, \\ P.Steinberg ${ }^{2}$, G.S.F.Stephans ${ }^{4}$, M.Stodulski ${ }^{3}$, A.Sukhanov ${ }^{2}$, J.-L.Tang ${ }^{5}$, R.Teng ${ }^{8}$, A.Trzupek ${ }^{3}$, C.Vale ${ }^{4}$, \\ G.J.van Nieuwenhuizen ${ }^{4}$, R.Verdier ${ }^{4}$, B.Wadsworth ${ }^{4}$, F.L.H.Wolfs ${ }^{8}$, B.Wosiek ${ }^{3}$, K.Woźniak ${ }^{3}$, A.H.Wuosmaa ${ }^{1}$, \\ B.Wysłouch ${ }^{4}$
}

1 Argonne National Laboratory, Argonne, IL 60439-4843, USA ${ }^{2}$ Brookhaven National Laboratory, Upton, NY 11973-5000, USA ${ }^{3}$ Institute of Nuclear Physics, Kraków, Poland ${ }^{4}$ Massachusetts Institute of Technology, Cambridge, MA 02139-4307, USA ${ }^{5}$ National Central University, Chung-Li, Taiwan ${ }^{6}$ University of Illinois at

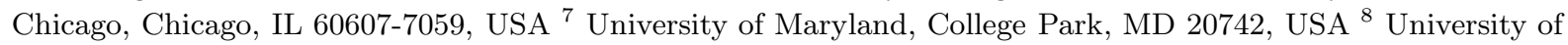
Rochester, Rochester, NY 14627, USA

The PHOBOS experiment at RHIC has measured the multiplicity of primary charged particles as a function of centrality and pseudorapidity in $\mathrm{Au}+\mathrm{Au}$ collisions at $\sqrt{s_{N N}}=19.6,130$ and $200 \mathrm{GeV}$. Two kinds of universal behavior are observed in charged particle production in heavy ion collisions. The first is that forward particle production, over a range of energies, follows a universal limiting curve with a non-trivial centrality dependence. The second arises from comparisons with $p p / \bar{p} p$ and $e^{+} e^{-}$data. $\left\langle N_{c h}\right\rangle /\left\langle N_{\text {part }} / 2\right\rangle$ in nuclear collisions at high energy scales with $\sqrt{s}$ in a similar way as $N_{c h}$ in $e^{+} e^{-}$collisions and has a very weak centrality dependence. This feature may be related to a reduction in the leading particle effect due to the multiple collisions suffered per participant in heavy ion collisions.

\section{Introduction}

The PHOBOS experiment has measured $d N_{c h} / d \eta$ and the average multiplicity of charged particles $\left\langle N_{c h}\right\rangle$ produced in heavy ion collisions for center of mass energies in the nucleon-nucleon center of mass system, $\sqrt{s_{N N}}$, of 19.6, 130 and $200 \mathrm{GeV}$. The data is also binned as a function of event centrality (impact parameter) characterized by the number of participating nucleons, $N_{\text {part }}$, allowing comparisons to elementary systems, like $p p / \bar{p} p$ and $e^{+} e^{-} \rightarrow$ hadrons.

The PHOBOS multiplicity detector consists of several arrays of silicon detectors which cover nearly the full solid angle for collision events. The event centrality is characterized by the multiplicity of charged particles measured by two sets of

\footnotetext{
*Current address: Physics Department, University of Cape Town, South Africa
}

16 paddle counters covering $3<|\eta|<4.5$. The methods used for measuring the multiplicity of charged particles as well as for extracting $N_{\text {part }}$ has been described in more detail in Ref. [1].

\section{Limiting Behavior in Pseudorapidity Distributions}

Fig. 11 shows $d N_{c h} / d \eta^{\prime} /\left\langle N_{\text {part }} / 2\right\rangle\left(\eta^{\prime}=\eta-\right.$ $\left.y_{\text {beam }}\right)$ measured at three different RHIC energies for peripheral $\left(\left\langle N_{\text {part }}\right\rangle \sim 100\right)$ and central events $\left(\left\langle N_{\text {part }}\right\rangle \sim 355\right)$, in the left and right panels, respectively. These show a clear "limiting behavior" in the fragmentation region. That is, the distributions are independent of beam energy in a substantial range in $\eta^{\prime}$. As the beam energy increases, $d N / d \eta^{\prime}$ follows the universal trend until it reaches $85-90 \%$ of it's maximum value at midrapidity, at which point it stops following the trend. Similar behavior has been observed in el- 
ementary collisions as well, both in $\bar{p} p$ collisions [2] and in $e^{+} e^{-}$collisions over a large range of energies [3].

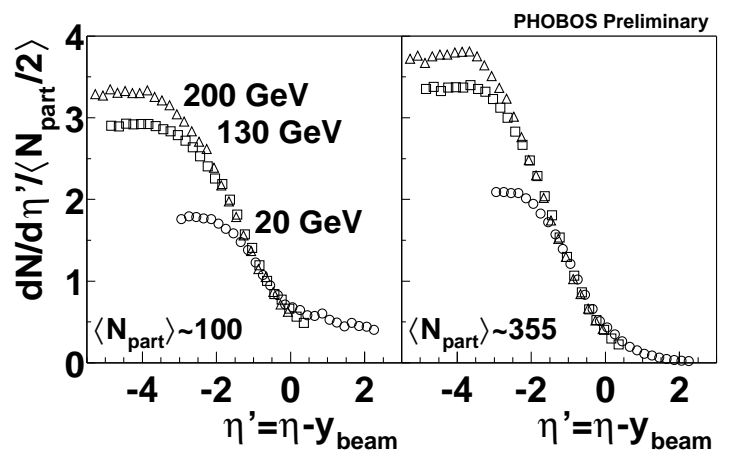

Figure 1. $d N / d \eta^{\prime} /\left\langle N_{\text {part }} / 2\right\rangle$ for peripheral $\left(\left\langle N_{\text {part }}\right\rangle \sim 100\right)$ and central $\left(\left\langle N_{\text {part }}\right\rangle \sim 355\right)$ events at three RHIC energies.

The limiting curve constrains the energy dependence of the charged particle multiplicity. It also varies with centrality in such a way that the increases seen at low $\eta^{\prime}$ (which is midrapidity in $\eta$ ) as $N_{\text {part }}$ increases, are accompanied by decreases near $\eta^{\prime} \sim 0$ (forward rapidities), as seen in Fig. 1. It is not clear why this behavior occurs, e.g. whether it is from energy conservation or a true long-range correlation.

\section{Comparison with Elementary Systems}

The angular distributions of charged particles for different strongly-interacting systems are shown in Fig. 2, where central $\mathrm{Au}+\mathrm{Au}$ (divided

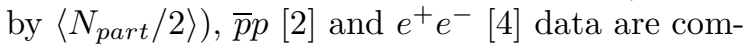
pared, all at $\sqrt{s}=200 \mathrm{GeV}$. In this figure, the $\mathrm{Au}+\mathrm{Au}$ and $\bar{p} p$ data are shown as $d N_{c h} / d \eta$, while the $e^{+} e^{-}$data (with cuts applied to reject events with large initial-state photon radiation) is shown as $d N / d y_{T}$, the rapidity distribution along the event thrust axis, calculated assuming the pion mass. The shapes of $\mathrm{Au}+\mathrm{Au}$ and $e^{+} e^{-}$are similar (within 10\%) in shape and magnitude, especially within $|\eta|<4$. In the lower panel, we also

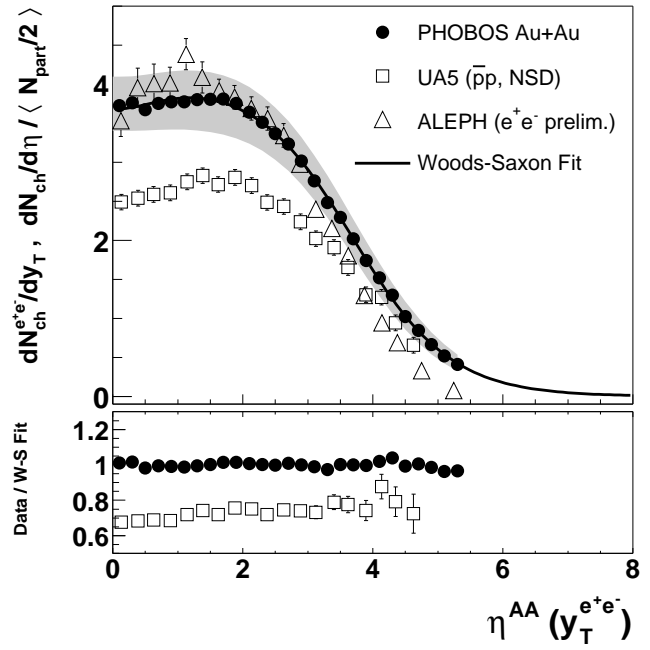

Figure 2. Top: $d N_{c h} / d \eta /\left\langle N_{\text {part }} / 2\right\rangle$ for central $\mathrm{Au}+\mathrm{Au}$ collisions at $\sqrt{s_{N N}}=200 \mathrm{GeV}$ compared with $\bar{p} p$ and $e^{+} e^{-}$data. Bottom: $\mathrm{Au}+\mathrm{Au}$ and $\bar{p} p$ data divided by a fit to the $\mathrm{Au}+\mathrm{Au}$ data.

observe that the shapes of the $\mathrm{Au}+\mathrm{Au}$ and $\bar{p} p$ distributions are also very similar over a large range in $\eta$, but the integrals differ by $\sim 40 \%$.

In Fig. 3, we compare $\left\langle N_{c h}\right\rangle /\left\langle N_{\text {part }} / 2\right\rangle$ in heavy ion collisions [5] to $e^{+} e^{-}$and $p p / \bar{p} p$ data over a large range in $\sqrt{s}$ [6]. It is observed that $\left\langle N_{c h}\right\rangle /\left\langle N_{\text {part }} / 2\right\rangle$ lies below $p p$ at low energies, passes through the $p p$ data around $\sqrt{s} \sim 10 \mathrm{GeV}$, and then gradually joins with the $e^{+} e^{-}$trend above CERN SPS energies. These comparisons can be seen more clearly by dividing all of the data by a fit to the $e^{+} e^{-}$data [7].

The $p p / \bar{p} p$ data follows the same trend as $e^{+} e^{-}$, but it can be shown that it matches very well if the "effective energy" $\sqrt{s}_{\text {eff }}=\sqrt{s} / 2$ is used, which accounts for the leading particle effect seen in $p p$ collisions [8]. Ref. [8] finds that bulk particle production in $p p$ and $e^{+} e^{-}$data does not depend in detail on the collision system but rather the energy available for particle production. In this scenario, the $\mathrm{Au}+\mathrm{Au}$ data suggests a substantially reduced leading particle effect in central collisions of heavy nuclei at high energy. 


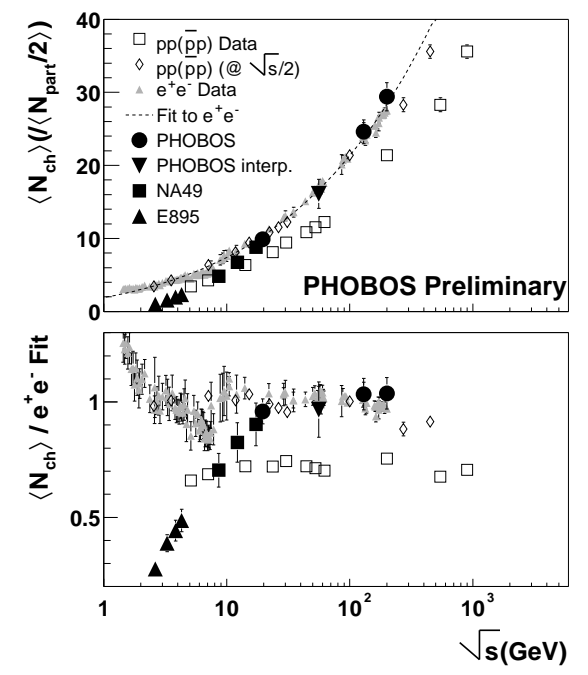

Figure 3. Comparison of $\left\langle N_{c h}\right\rangle /\left\langle N_{\text {part }} / 2\right\rangle$ for $\mathrm{A}+\mathrm{A}, p p / \bar{p} p$, and $e^{+} e^{-}$data compared with a fit to the $e^{+} e^{-}$data.

The alleviation of the leading particle effect might not be so surprising in nuclear collisions. Each participating nucleon is typically struck $\bar{\nu}=$ $\frac{N_{\text {coll }}}{N_{\text {part }} / 2}>3$ times on average as it passes through the oncoming gold nucleus for $N_{\text {part }}>65$. One could speculate that the multiple collisions transfer much more of the initial longitudinal energy into particle production. This naturally leads to the scaling of total particle production in heavy ion collisions with $N_{\text {part }}$, as seen in Fig. 局, reminiscent of the "wounded nucleon model" [9] but with the scaling factor determined by $e^{+} e^{-}$rather than $p p$.

In conclusion, PHOBOS has observed two kinds of universal behavior. The first is an energy-independent, but centrality-dependent, universal limiting distribution of charged particle production away from midrapidity. The second is that the total charged particle multiplicity per participant pair in heavy ion collisions above CERN SPS energies scales with sqrt(s) in a similar way as $e^{+} e^{-}$collisions. These features may offer a new perspective on particle production in heavy ion collisions.

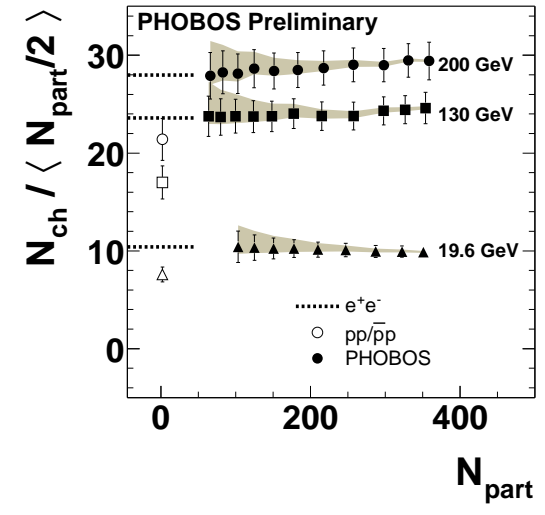

Figure 4. $\left\langle N_{c h}\right\rangle /\left\langle N_{\text {part }} / 2\right\rangle$ vs. $\quad N_{\text {part }}$ for $\sqrt{s_{N N}}=19.6,130$, and $200 \mathrm{GeV}$.

Special thanks to the ICHEP organizers and Heavy Ion convenors for the invitation to speak at the conference. This work was partially supported by U.S. DOE grants DE-AC02-98CH10886, DE-FG0293ER40802, DE-FC02-94ER40818, DE-FG02-94ER40865, DE-FG02-99ER41099, and W-31-109-ENG-38 as well as NSF grants 9603486, 9722606 and 0072204. The Polish groups were partially supported by KBN grant 2 PO3B 10323. The NCU group was partially supported by NSC of Taiwan under contract NSC 89-2112-M-008-024.

\section{REFERENCES}

1. B. B. Back et al., to be submitted to PRL.

2. G. J. Alner et al., Z. Phys. C 33, 1 (1986).

3. P. Abreu et al., Phys. Lett. B 459, 397 (1999).

4. H. Stenzel, ALEPH Collaboration, Contributed paper to ICHEP2000 (2000).

5. J. Klay, U.C. Davis PhD. Thesis (2001). S. V. Afanasiev et al., arXiv:nucl-ex/0205002 (2002).

6. D. E. Groom et al., Eur. Phys. J. C 15, 1 (2000).

7. A. H. Mueller, Nucl. Phys. B 213, 85 (1983).

8. M. Basile et al., Phys. Lett. B 92, 367 (1980). M. Basile et al., Phys. Lett. B 95, 311 (1980).

9. J. E. Elias et al. Phys. Rev. Lett. 41, 285 (1978). A. Białas, B. Bleszyński and W. Czyż, Nucl. Phys. B111, 461 (1976). 